\title{
Macrotendencias del talento humano y su comportamiento en las instituciones de educación superior en el Ecuador
}

Macrotendencias of human talent and its behavior in education institutions superior in Ecuador

Juan Carlos Castillo Moya. ${ }^{1}$, Marianela Bermejo Salmon. ${ }^{2}$, Ángel Bolívar Burbano Pérez. ${ }^{3}$ \& Raquel Ivon Flores León. ${ }^{4}$

Recibido: 28-04-2019 / Revisado: 25-05-209 /Aceptado: 25-06-2019/ Publicado: 25-07-2019

\begin{abstract}
DOI: https://doi.org/10.33262/cienciadigital.v3i3.2.753

The study was generated in some Institutions of Higher Education and its main objective was to analyze the macro-tendencies of human talent and their behavior from a systemic approach specifically in the Polytechnic Higher School of Chimborazo (ESPOCH) Ecuador. During the investigation, the combination of methods and techniques such as historical-logical as theoretical method, analysis and synthesis and induction and deduction, and empirical methods such as interviews, group work, direct observation, among others, were used. The results obtained made it possible to start from the argumentation of the theoretical and methodological foundations related to the macro tendencies of the human talent, the propensities of these and their adjustment to concrete conditions of the aforementioned institution could be shown and as a result the bases for the proposal of a plan of actions for its treatment in the studied context.
\end{abstract}

\footnotetext{
${ }^{1}$ Magister en Seguridad Industrial mención Prevención de Riesgos y Salud Ocupacional, Docente de la Escuela Superior Politécnica de Chimborazo, Ecuador, jrjdcastillo@gmail.com

${ }^{2}$ Doctora en Ciencias Económicas, Facultad de Ciencias Económicas y Empresariales, Universidad de Oriente, Ecuador,marianela@uo.edu.cu

${ }^{3}$ Ingeniero Industrial, Facultad de Administración de Empresas (FADE), Escuela Politécnica Superior de Chimborazo (ESPOCH), Ecuador, aburbano@espoch.edu.ec

${ }^{4}$ Psicóloga Educativa, Gobierno Autónomo Descentralizado Municipal Riobamba, Ecuador, flrakelivon.25@gmail.com
} 
Keywords: Human talent, macro talent trends, systemic approach

\section{Resumen}

El estudio se generó en algunas Instituciones de Educación Superior y su objetivo principal fue: analizar las macrotendencias del talento humano y su comportamiento desde un enfoque sistémico específicamente en la Escuela Superior Politécnica de Chimborazo (ESPOCH) de Ecuador. Durante la investigación se utilizó la combinación de métodos y técnicas tales como: métodos teóricos como el históricológico, análisis y síntesis e inducción y deducción y métodos empíricos como: entrevistas, trabajo grupal, observación directa entre otros. Los resultados obtenidos hicieron posible que partiendo de la argumentación de los fundamentos teóricos y metodológicos relativos a las macrotendencias del talento humano se pudieron mostrar las propensiones de estas y su ajuste a las condiciones concretas de la institución antes citada y en función de ello se aseveraron las bases para la propuesta de un plan de acciones para su tratamiento en el contexto objeto de estudio.

Palabras clave: Enfoque sistémico, macrotendencias del talento humano, talento humano.

\section{Introducción:}

El ritmo acelerado de los cambios que se vienen operando en la contemporaneidad en el orden tecnológico, informativo y de actuación del hombre insertado en las organizaciones desde un enfoque direccionado en cómo gestionar recursos, tiempo y personas en función de la consecución de determinados objetivos enmarcados en la era del conocimiento y la innovación, ha incidido y continuará incidiendo interiormente en las Instituciones de Educación Superior en cualquier contexto, por cuanto, estas no solo se enfrentan a innovaciones en las Ciencias y en la Tecnología, sino también a las modificaciones en los principios esencialmente pedagógicos acordes a conceptos relativos a las competencias y profesionalismo que deben caracterizar al hombre en la actualidad. Las consecuencias serán, inevitablemente, el surgimiento de cambios en el funcionamiento de estas instituciones y en tal sentido se tiene que: las estructuras más antiguas y obsoletas de las mismas están cediendo terreno ante los sistemas actuales; más novedosos y más armónicos sustentados en las leyes, políticas y normativas vigentes específicas de cada ámbito de actuación; en el caso de Ecuador se destaca el ajuste al Plan Nacional del Buen Vivir vigente hasta el 2019 y el Plan 
Nacional de Desarrollo para Toda Una Vida puesto que se pondrá en práctica a partir del 2020.

Resulta pues evidente que el talento humano, como elemento que forma parte de las organizaciones y que está presente en todos los subsistemas de estas y que sin duda, constituye un factor esencial, no ha sido considerado de la misma forma a lo largo del siglo anterior y aún en algunos casos durante el presente siglo, y su importancia tampoco ha sido vista de igual modo por los distintos teóricos y prácticos que de él se han ocupado tanto desde su actuación interna como externa; por lo que ha sido tratado en muchos casos desde una posición pasiva y reactiva y no en su naturaleza activa y proactiva enmarcado en un enfoque de gestión, atemperado en su comportamiento en el entorno en que se enmarcan las instituciones donde estos se insertan primeramente desde su ámbito interno contextualizado en correspondencia con las áreas de resultados clave y de apoyo que lo conforman. Y donde es este talento quien realiza las diferentes tareas y funciones que le competen, bien cuando actúa como un agente de línea o cuando su rol está más bien desde el asesoramiento de los procesos que tienen lugar en estas instancias.

La Gestión del Talento es un término indubitablemente ligado a la Administración y esta, requiere de nuevas ideas, y para conseguirlas, se demanda de una formación conceptual y metodológica, así como de enorme calidad académica la cual solo puede ser obtenida por medio del capital humano del que disponen las universidades las que, por su parte, se encuentran centradas en dar cumplimiento a las políticas públicas que se establecen para las universidades (Orizaga, 2011).

(Cardona Andújar, 2011) "Con el término gestión hacemos referencia aquí al conjunto de acciones didáctico-organizativas necesarias para lograr una meta final determinada centrada en la construcción, transferencia y adquisición de conocimiento en la totalidad de las áreas de formación”. Las Instituciones de Educación Superior del Ecuador cualquiera que sea el contexto donde estas se insertan requieren de condiciones que permitan gestionar su talento humano en función de garantizar la calidad de los procesos sustantivos que en estas tienen lugar tales como la docencia, investigación, vinculación y gestión. Es importante entonces que el hombre desarrolle al máximo su talento, expresado en lo esencial en su poder creativo que les posibilite afrontar las transformaciones que en el orden teórico, metodológico y práctico se requieren desde las investigaciones científicas en los Centros de Educación 
Superior.

De ahí, la necesidad de realizar investigaciones con un rigor científico, que permitan ante todo conocer las orígenes, revelaciones y permisibles acciones que puedan emprenderse durante el período 2017 y 2018 en el comportamiento del talento humano.

Por lo que el objetivo de este estudio está dado por: analizar las macrotendencias del talento humano y su comportamiento desde un enfoque sistémico específicamente en la Escuela Superior Politécnica de Chimborazo (ESPOCH) de Ecuador.

\section{Metodología:}

Durante la realización de la investigación se empleó desde el enfoque de la Economía Política el método del Materialismo Dialéctico e Histórico, en su acepción más precisa con la concepción materialista de la historia que comprende los métodos siguientes:

Histórico - Lógico: fue empleado para la descripción y el comportamiento desde una secuencia lógica de las macrotendencias del talento humano y su evolución.

Análisis y Síntesis: se utilizó durante todas las fases del trabajo, en el procesamiento de la bibliografía consultada y su análisis crítico.

Métodos empíricos: fueron aplicadas las técnicas utilizadas en el desarrollo de la investigación, tales como:

> Entrevistas: para conocer los estados de opinión respecto a cómo se manifiestan las tendencias antes referenciadas en las Instituciones de Educación Superior del Ecuador.

> Observación directa: en la comprobación del comportamiento real de las macrotendencias desde las áreas de resultados clave y de apoyo.

Trabajo grupal: para el tratamiento de las macrotendencias con la finalidad de encontrar manifestaciones consensuales sobre estas.

\section{Resultados:}

Primeramente, se enunciarán las macrotendencias del talento humano de referencia, y resaltan aspectos importantes tal y como se refieren en (Chiavenato, 2010) tales como: 
Tabla 1. Comportamiento de las macrotendencias del talento humano

\begin{tabular}{ll}
\hline Macrotendencias del talento humano & Tendencia actual \\
\hline Una nueva filosofía de acción. & En todos los niveles de la organización, las personas se \\
& consideran socias que conducen los negocios de la empresa, \\
& utilizan la información disponible, aplican sus conocimientos y \\
& habilidades y toman las decisiones adecuadas para garantizar los \\
& resultados esperados
\end{tabular}

Nítida y rápida tendencia al downsizing. EI área de recursos humanos (ARH) se está reestructurando, reduciendo y comprimiendo hasta el nivel esencial o básico. Con esta reducción, los ejecutivos de recursos humanos están cambiando radicalmente sus atribuciones.

Transformación de un área de servicios en un área de consultoría interna.

Transferencia gradual de decisiones y acciones de la ARH hacia la gerencia de línea.

Intensa conexión con el negocio de la empresa.

Énfasis en la cultura participativa y democrática en las organizaciones

Utilización de mecanismos de motivación y de realización personal

Adecuación de las prácticas y políticas de $\mathrm{RH}$ a las diferencias individuales de las personas.

Viraje completo en dirección a/ cliente, sea interno o externo.

Gran preocupación por /a creación de valor en la empresa.

Preocupación por preparar la empresa y a las personas para e/ futuro.

Utilización intensiva del benchmarking como estrategia de mejoramiento continuo de los procesos y servicios.
La antigua organización funcional está dando lugar a la organización por procesos. En vez de órganos o departamentos, el ARH está coordinando procesos o subsistemas.

Es el traslado de actividades, antes centradas en el ARH, a los gerentes de las demás áreas de la empresa. Esto es lo esencial: la búsqueda de la eficacia y de la excelencia a partir de las personas.

El ARH se está desplazando hacia la planeación estratégica de la empresa, y está desarrollando medios que permitan a las personas caminar hacia los objetivos organizacionales de manera proactiva.

EI área está perdiendo su antiguo aire severo y sombrío, para ganar una nueva posturaabierta, amigable y de apoyo y soporte a las personas.

Se están destacando y valorando los objetivos y las necesidades individuales de las personas.

Las personas son realzadas como personas y no como recursos productivos.

La antigua tendencia a la estandarización de reglas y procedimientos está cediendo lugar a las prácticas alternativas diseñadas de acuerdo con los deseos y necesidades individuales delos empleados.

La ARH se está orientando hacia el usuario. Los gerentes y empleados se están orientando hacia la satisfacción de los clientes.

Creación de valor para el cliente o, incluso, aumento de valor para el contribuyente. El ARH tiene mucho que ver con la educación de las personas y con la toma de concienciaorientada a generar valor en la organización continuamente.

El ARH está abandonando su comportamiento pasivo y reactivo para adoptar una posición proactiva y orientada al futuro para anticiparse a las exigencias y necesidades de la organización.

EI benchmarking refleja una visión orientada hacia el ambiente externo y hacia lo que existe de excelencia allí. EI benchmarking refleja una visión orientada hacia el ambiente externo y hacia lo que existe de excelencia allí. 
ISSN: 2602-8085

WWW.cienciadigital.org

Vol. 3, $\mathrm{N}^{\circ} 3.2$, p. 266-276, julio - septiembre, 2019

Caracterización de las tendencias actuales del talento humano contexto en que se desarrolla este estudio (adaptado de Chiavenato, 2010).

En esta dirección del estudio se parte de ajustar los fundamentos teóricos que conforman las macrotendencias antes referenciadas al ámbito en que fueron tratadas las mismas o sea a las manifestaciones atribuibles a estas en la ESPOCH, con el empleo de la observación directa, la aplicación de entrevistas, la deducción e inducción de situaciones y el análisis y síntesis del contenido abordado tal y como sigue:

\section{Nueva filosofía de acción}

Considerar a las personas como seres humanos y no simples recursos empresariales donde un factor clave son sus competencias vinculadas a los procesos donde estas se gestan desde posiciones proactivas, ejemplo de ello: la inexistencia de perfiles de cargo por competencias con un enfoque sistémico extensiva a la carencia de una alineación en tal sentido de los procesos que conforman la gestión del talento humano conducen a que; aún no se comienza por satisfacer las necesidades y expectativas del cliente interno, ello significa no promover una nueva mentalidad, o sea un cambio en la forma de pensar y actuar y su ineludible efecto en el ámbitofundamentalmente económico y social.

\section{Nítida y rápida tendencia al downsizing}

No obstante aunque se han experimentado algunos avances en el rol del área de recursos humanos en la institución en correspondencias con las tendencias actuales de esta en el mundo, dígase, énfasis en su naturaleza de área de apoyo a las áreas de resultados clave y asesor de los procesos relativos en materia laboral desde una posición activa , aún la misma ni estructural, ni funcionalmente está integrada a estas áreas sino que en ella se sigue aglutinando todos sus subsistemas y desempeña en este sentido lo antes expuesto. Se reafirma lo expuesto por (Chiavenato, 2010, p8) "el downsizing es más un ajuste para los problemas actuales, que una directriz para la empresa del mañana. Sirve como correctivo para ajustar las operaciones del pasado a la realidad de hoy, pero no constituye una ruta que oriente el futuro del área; solo pone la casa en orden. Pero esto es lo que las organizaciones están haciendo".

\section{Transformación de un área de servicios en un área de consultoría interna}

Aunque se gestiona por el Área de Recursos Humanos (ARH) la consultoría interna de todos los procesos o subsistemas de la gestión del talento humano, se necesita de una coordinación en su integridad de los procesos, subprocesos que conduzca a la salida que esto demanda; un producto de excelencia orientado a los resultados finales de la institución que satisfaga las necesidades y expectativas de sus clientes y de la sociedad en su generalidad.

4. Transferencia gradual de decisiones y acciones de la ARH hacia la gerencia de línea Si se parte de la estructura organizativa de las IES y su descentralización en facultades y 
estas en departamentos y áreas, puede apreciarse un paulatino avance en que las decisiones sobre política laboral se gestionen por estas y no centralizadamente en tal sentido cada facultad o departamento o áreas dentro de estos, están facultadas para tomar decisiones sobre selección, capacitación, desempeño y a tales efectos los directivos de línea se convierten en gestores de personas que no administran a estas, sino que interactúan con las mismas, asesorados por los gestores del talento humano a estas instancias, lográndose un nexo entre el área de recursos humanos en toda la institución y sus dependencias en las direcciones antes señaladas.

\section{Intensa conexión con la misión de la Institución}

El ARH se está desplazando hacia la planeación estratégica de la institución o sea la planeación de los Recursos Humanos se vincula con la planeación estratégica de la institución para que sus planes apoyen y estimulen la misión de la organización a través de los ajustes frecuentes necesarios, de la oferta y demanda del personal en un contexto en constante cambio. Pero desde su propia génesis hasta su ejecución por el colectivo humano su actuar inmediato se enmarca con una visión estratégica más en el corto y en el mediano plazo, que en el largo plazo y no se remonta solo en el tiempo, sino también en concebir acciones estratégicas que promuevan un distinguido impacto social

\section{6.Énfasis en la cultura participativa y democrática en las organizaciones}

La administración consultiva y participativa con las personas que permite a estas trabajar en una cultura democrática e impulsora. Existe una preocupación por el clima organizacional y la satisfacción de las personas, pero con marcado énfasis en las implicaciones internas que en aquellas que trascienden el marco de la organización y se proyecten hacia la solución de problemas del entorno social en que estas se desenvuelven.

\section{Utilización de mecanismos motivación y realización del personal}

Aun cuando resalten y se estén valorando los objetivos y las necesidades individuales de las personas, es ineludible que se confrontan problemas en la búsqueda de los medios para ofrecer oportunidades de realización personal plena de los empleados, que les permita sentirse motivados por ejemplo a través de una proyección adecuada de la capacitación y desarrollo, la aplicación de evaluación del desempeño en función de las competencias laborales, la aplicación del sistema de pago por resultados que se corresponda en su generalidad con las funciones que el personal implicado desempeña y sus consabidas implicaciones económicas y sociales.

\section{Adecuación de las prácticas y políticas de RH (recurso humano) a las diferencias individuales de las personas}

No obstante a que se han logrado algunos avances en el establecimiento de las individualidades del personal y en correspondencia con ello perfilar y un programa de 
ISSN: 2602-8085

WWW.cienciadigital.org

Vol. 3, N³.2, p. 266-276, julio - septiembre, 2019

acciones tendientes a satisfacer sus necesidades mínimas y esenciales y en esta dirección atenuar los efectos desfavorables de antiguas políticas de estandarización de reglas y procedimientos inflexibles; el no tener bien definidos sus perfiles de cargo y las competencias laborales, por proceso y organizacionales desfavorece el avance acreciente de diseñar por una parte y de lograr por otra perfeccionar acciones concretas de acuerdo con los deseos y necesidades individuales de los empleados.

\section{Viraje completo en dirección a/ cliente, sea interno o externo}

Se están gestando políticas orientadas tanto al cliente interno como externo con el fortalecimiento de un trabajo mancomunado tanto desde lo teórico, dígase ; diseños de políticas portadoras de suficientes acciones que en la práctica satisfagan las necesidades de los usuarios a tales efectos se ha ampliad la cartera de productos que pueden resumirse entre otras en asesorías, entrenamientos direccionadas al logro de la interrelación entre los intereses personales y los institucionales y por tanto en la atracción cada vez más de los consumidores del producto ofertado.

\section{Gran preocupación por la creación de valor en la organización}

Aunque es de interés de la alta dirección de la institución la consecución continua de buenos resultados donde prevalezca añadir valor al servicio ofertado esto se contrarresta por las insuficiencias y deficiencias experimentadas en el desempeño del ARH que no detecta a tiempo en ocasiones en qué momento es necesaria la capacitación y para qué persona en concreto se debe ofrecer, sin obviar e insistir siempre en los trabajadores que lo más importante para todos es el cumplimiento de los objetivos tanto del cliente interno como externo y si para ello es necesario aprender algo nuevo, se debe hacer.

\section{Preocupación por preparar a la institución y a las personas para el futuro}

Aún se mantiene en algunos procesos un comportamiento pasivo y reactivo limitado al control en los casos que procede de asistencia, puntualidad, preparación de nóminas para el pago del salario y no su rol orientador y naturaleza asesora de los procesos que tienen lugar en la institución y su repercusión para la sociedad por ejemplo; métodos de selección poco apropiados; en ocasiones, no se tiene en cuenta los procedimientos establecidos para la selección del personal acorde a las normativas vigente para las IES y en tal sentido los facultados para realizar el reclutamiento y la selección no poseen todas las habilidades para detectar a las personas adecuadas para cada posición, lo que aumenta el riesgo de equivocarse, algo que, aunque siempre existe, se debe intentar minimizar.

Utilización intensiva del benchmarking como estrategia de mejoramiento continuo de los procesos y servicios.

Aunque se promueven acciones para mejorar los servicios, es incipiente, prácticamente nula la utilización del benchmarking por el ARH. 
El tratamiento de las macrotendencias antes citadas y los resultados obtenidos, sugieren la propuesta de un programa de acciones que permitan atenuar los puntos débiles y/o vulnerables que estas presentan, tal y como se muestra seguidamente:

Tabla 2. Programa de acciones

\begin{tabular}{|c|c|c|c|c|}
\hline Macrotendencias & Punto Débil & Criterio de Medidas & Responsable & $\begin{array}{c}\text { Fecha de } \\
\text { Cumplimiento }\end{array}$ \\
\hline \multirow[t]{2}{*}{$\begin{array}{l}\text { Nueva filosofía de } \\
\text { acción }\end{array}$} & $\begin{array}{l}\text { Inexistencia de } \\
\text { perfiles de cargo } \\
\text { por competencias } \\
\text { con un enfoque } \\
\text { sistémico. }\end{array}$ & $\begin{array}{l}\text { Elaborar los perfiles de cargo por } \\
\text { competencias que permitan conocer } \\
\text { las funciones y requerimientos del } \\
\text { personal y en función de ello; } \\
\text { identificar las competencias } \\
\text { requeridas por los puestos de } \\
\text { trabajo. }\end{array}$ & Jefe de ARH & Diciembre 2019 \\
\hline & $\begin{array}{l}\text { Carencia de una } \\
\text { alineación en tal } \\
\text { sentido de los } \\
\text { procesos que } \\
\text { conforman la } \\
\text { gestión del talento } \\
\text { humano. }\end{array}$ & $\begin{array}{l}\text { Incorporar como necesidades de } \\
\text { aprendizajes; procedimientos, } \\
\text { metodologías que sienten las bases } \\
\text { para lograr una alineación entre los } \\
\text { procesos de gestión del } \\
\text { talento humano. }\end{array}$ & Jefe de ARH & $\begin{array}{l}\text { Septiembre- } \\
\text { diciembre } 2019 \\
\text { Octubre- } \\
\text { diciembre } 2019\end{array}$ \\
\hline $\begin{array}{l}\text { Transformación de } \\
\text { un área de } \\
\text { servicios en un } \\
\text { área de consultoría } \\
\text { interna }\end{array}$ & $\begin{array}{l}\text { Falta de una } \\
\text { coordinación en su } \\
\text { integridad de los } \\
\text { procesos, } \\
\text { subprocesos que } \\
\text { conduzca a la salida } \\
\text { de un producto de } \\
\text { excelencia. }\end{array}$ & $\begin{array}{l}\text { Plan de preparación que garantice la } \\
\text { adecuada interacción entre la } \\
\text { generación de nuevo conocimientos y } \\
\text { los servicios ofertados en función de } \\
\text { la integración de } \\
\text { estos. }\end{array}$ & Jefe de ARH & $\begin{array}{l}\text { En cada } \\
\text { trimestre } \\
\text { de } 2019\end{array}$ \\
\hline $\begin{array}{l}\text { Énfasis en la } \\
\text { cultura } \\
\text { participativa y } \\
\text { democrática en las } \\
\text { organizaciones. }\end{array}$ & $\begin{array}{l}\text { Énfasis } \\
\text { mayormente en las } \\
\text { implicaciones } \\
\text { internas asociadas } \\
\text { al clima } \\
\text { organizacional y la } \\
\text { satisfacción } \\
\text { de las personas. }\end{array}$ & $\begin{array}{l}\text { Evaluar el sistemáticamente el } \\
\text { comportamiento de los factores que } \\
\text { inciden en el clima organizacional del } \\
\text { personal y su } \\
\text { incidencia en la sociedad. }\end{array}$ & Jefe de ARH & $\begin{array}{l}\text { En cada } \\
\text { trimestre } \\
\text { de } 2019\end{array}$ \\
\hline $\begin{array}{l}\text { Utilización de } \\
\text { mecanismos } \\
\text { motivación y } \\
\text { realización del } \\
\text { personal. }\end{array}$ & $\begin{array}{l}\text { Insuficiencias en la } \\
\text { búsqueda de los } \\
\text { medios para ofrecer } \\
\text { oportunidades de } \\
\text { realización plena de } \\
\text { los empleados, que } \\
\text { les permita sentirse } \\
\text { motivados. }\end{array}$ & $\begin{array}{l}\text { Emprender acciones } \\
\text { que permitan la realización del talento } \\
\text { humano y evaluar su impacto en la } \\
\text { satisfacción y expectativas de estos. }\end{array}$ & Jefe de ARH & $\begin{array}{l}\text { Trimestral y } \\
\text { anualmente } \\
\text { durante el } 2019\end{array}$ \\
\hline $\begin{array}{l}\text { Preocupación por } \\
\text { preparar a la } \\
\text { institución y a las } \\
\text { personas para el } \\
\text { futuro. }\end{array}$ & $\begin{array}{l}\text { Carácter pasivo y } \\
\text { reactivo de los } \\
\text { enfoques } \\
\text { tradicionales del } \\
\text { talento humano en } \\
\text { algunos procesos de } \\
\text { ARH. }\end{array}$ & $\begin{array}{l}\text { Gestionar la aplicación de mecanismos } \\
\text { que permitan el accionar desde una } \\
\text { posición activa y proactiva del talento } \\
\text { humano en los procesos de selección } \\
\text { del personal y evaluación del } \\
\text { desempeño entre } \\
\text { otros. }\end{array}$ & Jefe de ARH & $\begin{array}{l}\text { Trimestral y } \\
\text { anualmente } \\
\text { durante el } 2019\end{array}$ \\
\hline
\end{tabular}




\section{Conclusiones:}

Del estudio efectuado hizo posible resaltar los aspectos siguientes:

- El análisis de las 12 macrotendencias del talento humano permitió comprobar en la práctica el comportamiento de las mismas en el contexto seleccionado, y que su naturaleza no es casual y ni debe ser inestable a fin de perfeccionar de la gestión del talento humano y de la organización.

- Se demostró la necesidad de ajustar desde lo externo e interno las tendencias del talento humano en las Instituciones de Educación Superior, dado que ello permite avanzar al logro de su competitividad y excelencia en medio del entorno turbulento en que estas se desarrollan.

- El estudio y análisis de las macrotendencias expuestas propició las bases para la proyección de posibles soluciones en aquellas vinculadas mayormente con la actuación del talento humano insertado en el ARH.

- Como resultado de la investigación realizada se dispone de un soporte informativo que impacta positivamente en lo fundamental en el orden teórico, metodológico, económico y social en el contexto analizado.

\section{BIBLIOGRAFIA}

- CARDONA ANDÚJAR, J. 2010. Hacia un modelo para la gestión del talento humano en la enseñanza universitaria. Aspectos básicos Artículos Facultades de Pedagogía. Pampedia, pp. $31-43$

- CHIAVENATO, I. 2010. La gestión del talento humano. Las macrotendencias de la gestión del talento humano.

- CHIAVENATO, I. 2012. Administración de Recursos Humanos, México, Editorial Mc Graw Hill.

- CUESTA SANTOS, A. 2010. Tecnología de Gestión de Recursos Humanos, La Habana, Editorial "Félix Varela.

- DÍAZ GISPERT, L. 2007. La Gestión de los Recursos Humanos y su evolución con el desarrollo de la administración en Cuba, Cuba.

- ORIZAGA RODRÍGUEZ, C. 2011. Gestión del Talento Humano. Revista El Buzón de Pacioli [Online]. Available: www.itson.mx/pacioli.

- SAlAZAR, D. 2012. La Red Capital Humano, La Habana, Cuba, Editorial Academia.

- SOLTURA, A. 2007. Tecnología para la determinación de los perfiles de cargo por competencias en las organizaciones cubanas. Tesis de maestría inédita, Instituto Superior Politécnico José Antonio Echeverría. 


\section{PARA CITAR EL ARTÍCULO INDEXADO.}

Castillo Moya, J., Bermejo Salmon, M., Burbano Pérez, Ángel, \& Flores León, R. (2019). Macrotendencias del talento humano y su comportamiento en las instituciones de educación superior en el Ecuador. Ciencia Digital, 3(3.2), 266-276. https://doi.org/10.33262/cienciadigital.v3i3.2.753

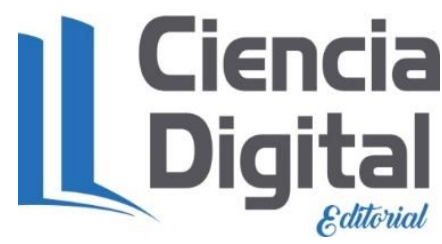

El artículo que se publica es de exclusiva responsabilidad de los autores y no necesariamente reflejan el pensamiento de la Revista Ciencia Digital.

El artículo queda en propiedad de la revista y, por tanto, su publicación parcial y/o total en otro medio tiene que ser autorizado por el director de la Revista Ciencia Digital.
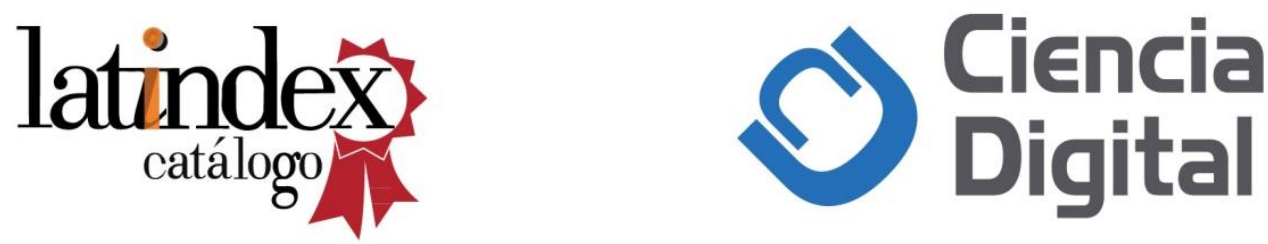